\title{
Enhanced expression of BMP6 inhibits hepatic fibrosis in non-alcoholic fatty liver disease
}

\author{
Stephanie Arndt, ${ }^{1}$ Eva Wacker, ${ }_{1}^{1}$ Christoph Dorn, ${ }^{2}$ Andreas Koch, ${ }^{2}$ Michael Saugspier, ${ }^{2}$ \\ Wolfgang E Thasler, ${ }^{3}$ Arndt Hartmann, ${ }^{4}$ Anja Katrin Bosserhoff, ${ }_{1}^{1}$ Claus Hellerbrand ${ }^{2}$
}

\begin{abstract}
- Additional material is published online only. To view please visit the journal online (http://dx.doi.org/10.1136/ gutjnl-2014-306968).

${ }^{1}$ Institute of Pathology, University Regensburg, Regensburg, Germany ${ }^{2}$ Department of Internal Medicine I, University Hospital Regensburg, Regensburg, Germany

Grosshadern Tissue Bank and Center for Liver Cell Research, Department of Surgery, Ludwig-Maximilians-University Munich, Munich, Germany ${ }^{4}$ Institute of Pathology, University Hospital Erlangen, Erlangen, Germany
\end{abstract}

\section{Correspondence to} Professor Claus Hellerbrand, Department of Internal Medicine I, University Hospital Regensburg, Franz-JosefStrauss-Allee 11, Regensburg D-93053, Germany; claus.hellerbrand@ukr.de

$\mathrm{AKB}$ and $\mathrm{CH}$ are senior authors contributed equally.

Received 7 February 2014 Revised 19 June 2014 Accepted 23 June 2014 Published Online First 10 July 2014

\section{ABSTRACT}

Objective Bone morphogenetic protein 6 (BMP6) has been identified as crucial regulator of iron homeostasis. However, its further role in liver pathology including non-alcoholic fatty liver disease (NAFLD) and its advanced form non-alcoholic steatohepatitis (NASH) is elusive. The aim of this study was to investigate the expression and function of BMP6 in chronic liver disease. Design BMP6 was analysed in hepatic samples from murine models of chronic liver injury and patients with chronic liver diseases. Furthermore, a tissue microarray comprising 110 human liver tissues with different degree of steatosis and inflammation was assessed. BMP6deficient $\left(\mathrm{BMP}^{-l-}\right.$ ) and wild-type mice were compared in two dietary NASH-models, that is, methionine cholinedeficient (MCD) and high-fat (HF) diets.

Results BMP6 was solely upregulated in NAFLD but not in other murine liver injury models or diseased human livers. In NAFLD, BMP6 expression correlated with hepatic steatosis but not with inflammation or hepatocellular damage. Also, in vitro cellular lipid accumulation in primary human hepatocytes induced increased BMP6 expression. MCD and HF diets caused more hepatic inflammation and fibrosis in $\mathrm{BMP}^{-/-}$ compared with wild-type mice. However, only in the MCD and not in the HF diet model BMP6 ${ }^{-1-}$ mice developed marked hepatic iron overload, suggesting that further mechanisms are responsible for protective BMP6 effect. In vitro analysis revealed that recombinant BMP6 inhibited the activation of hepatic stellate cells (HSCs) and reduced proinflammatory and profibrogenic gene expression in already activated HSCS.

Conclusions Steatosis-induced upregulation of BMP6 in NAFLD is hepatoprotective. Induction of BMP6signalling may be a promising antifibrogenic strategy.

\section{INTRODUCTION}

Bone morphogenetic proteins (BMPs) are multifunctional growth factors belonging to the transforming growth factor $\beta$ (TGF- $\beta$ ) superfamily. The BMP family consists of more than 30 members. In humans, 19 BMP members are under the designation of BMPs, and according to their gene homology, protein structure and functions, they are further subdivided into at least four subgroups: BMP2/4, BMP5/ 6/7/8a/8b, BMP9/10 and BMP12/13/14. ${ }^{1}$

Although BMPs were initially identified as osteogenic factors present in demineralised bone capable of inducing ectopic bone formation, it is now evident that BMPs perform several other functions during embryonic development as well as in adult tissue

\section{Significance of this study}

What is already known on this subject?

- BMP6 is a critical regulator of iron homeostasis.

- Disruption of iron homeostasis is a putative element inducing liver damage and fibrosis in non-alcoholic fatty liver disease (NAFLD).

What are the new findings?

- Hepatic BMP6 expression is increased in murine and human NAFLD but not in other chronic liver diseases.

- Hepatocellular lipid accumulation induces BMP6 expression in murine and human hepatocytes.

- BMP6 protects from non-alcoholic steatohepatitis (NASH) in two independent mouse models.

- Effects on iron homeostasis are only partially responsible for the hepatoprotective BMP6 effect in NAFLD in mice.

- BMP6 directly inhibits anti-inflammatory and antifibrogenic effects on murine and human hepatic stellate cells.

How might it impact on clinical practice in the foreseeable future?

- Induction of BMP6 signalling appears as a potential therapeutic option for the prevention and treatment of NASH.

- BMP6 expression levels may have potential as predictive markers for the progression of NAFLD.

regeneration and homeostasis. More recently, a role for BMPs in different human diseases has started being revealed. Similar to other TGF- $\beta$ family members, such as TGF- $\beta 1$, both tumour suppressor and tumour promoter activities have been described for BMPs, depending on various factors such as the dose and the type of the target cell. ${ }^{2}$

We found enhanced levels of several BMPs (BMP4, 6, 7, 8, 9, 10, 11, 13 and 15) in hepatocellular carcinoma (HCC) compared with normal liver tissues $^{3}$ and demonstrated that general BMP inhibition decreased the invasive phenotype of HCC cells in vitro. In line with this, we confirmed enhanced BMP4 expression and its correlation with aggressive tumour growth in human HCC tissues. ${ }^{4}$ Most recently, $\mathrm{Li}$ et $a l^{5}$ identified that enhanced 
BMP9 expression induces epithelial to mesenchymal transition and herewith invasiveness of HCC cells in vitro. Besides HCC, Nakatsuka et $a l^{6}$ reported transient expression of BMP2 and BMP4 in acute liver injury. The same group demonstrated the critical role of BMP signalling in the wound healing response in acute liver injury using mice with conditional knockout of Bmpr1a, a critical receptor for BMP2 and BMP4 signal transduction. ${ }^{7}$

In chronic liver disease, TGF- $\beta$ is well known to play a central role in liver fibrosis, but surprisingly little is known about the role of BMPs. Present studies focused on BMP7 and here, the BMP signalling role in liver fibrosis appears controversial. ${ }^{8}$ Hepatic BMP7 expression is upregulated in adults with cirrhosis and in children with biliary atresia, and elevated BMP7 concentration in the serum was concomitant with the progression of liver fibrosis. ${ }^{9}{ }^{10}$ Contrary, administration of BMP7 inhibits progression of fibrosis in experimental models in mice and rats. ${ }^{11} 12$

BMP6 belongs to the same BMP subfamily as BMP5 and BMP7. Several studies demonstrated that BMP6 plays a central role in the control of iron homeostasis. Andriopoulos et al ${ }^{13}$ and Meynard et al ${ }^{14}$ identified BMP6 as an important endogenous regulator of the expression of hepcidin, a protein that is mainly expressed in hepatocytes and controls the iron entry into the plasma. Both groups demonstrated that BMP6-deficient mice develop massive iron overload, and in a previous study we added essential and complementary information to these two reports. ${ }^{15}$ Most importantly, we discovered that BMP6 is produced upon iron exposure predominantly in the small intestine. ${ }^{15}$ Still, BMP6 is also expressed in liver cells, ${ }^{16}$ and functional studies suggest a role for hepatocyte-derived BMP6 in the control of hepcidin expression levels. ${ }^{17}$ Notwithstanding these significant observations, the role of BMP6 in liver pathology remained elusive.

The aim of this study was to study the expression and function of BMP6 in chronic liver diseases.

\section{MATERIALS AND METHODS \\ Cells and cell culture}

Primary murine and human hepatocytes, hepatic stellate cells (HSCs) and Kupffer cells were isolated and cultured as described. ${ }^{18} 19$ In vitro activation of HSCs was achieved by cell culture on uncoated tissue culture dishes. ${ }^{18}$ For individual experiments, cells were incubated with recombinant BMP6 $(10,50,100$ or $200 \mathrm{ng} / \mathrm{mL})$ as indicated or with tumour necrosis factor- $\alpha$ (TNF- $\alpha, 10 \mathrm{ng} / \mathrm{mL}$ ) (both from R\&D Systems, Wiesbaden-Nordenstadt, Germany). Furthermore, cellular lipid accumulation in human and murine hepatocytes was induced by incubation with free fatty acids applying an in vitro model that we have recently described. ${ }^{20}$

\section{Human tissues and tissue microarray}

A tissue microarray (TMA) was constructed out of 110 paraffin-embedded human hepatic tissue specimens with different degree of steatosis and inflammation. ${ }^{21}$ All cases were reviewed by a surgical pathologist (AH). A modified histological non-alcoholic fatty liver disease (NAFLD)/non-alcoholic steatohepatitis (NASH) score according to the recommendations of the Nonalcoholic Steatohepatitis Clinical Research Network was used. ${ }^{22}$

In addition to formalin-fixed liver tissues for TMA construction, liver specimens of patients with NAFLD $(n=12)$ and patients with cirrhosis $(n=15)$, and normal human liver tissues $(n=8)$, which had been immediately snap frozen after surgical resection and stored at $-80^{\circ} \mathrm{C}$, had been available.
Human liver tissues were obtained and experimental procedures were performed according to the guidelines of the charitable state controlled foundation Human Tissue and Cell Research (HTCR), with the informed patients' consent, and the study was approved by the local ethics committee of the University Regensburg.

\section{Mice and murine models of hepatic steatosis, inflammation and fibrosis}

129Sv/Ev wild-type (wt) $\left(\mathrm{BMP}^{+/+}\right)$and $\mathrm{BMP}^{-/-}$mice on 129Sv/Ev inbred background were obtained from the Robertson laboratory (Department of Molecular and Cellular Biology, Harvard University, Cambridge, Massachusetts, USA). ${ }^{23}$ Male wt and $\mathrm{BMP}^{-/-}$were exposed to two different dietary NASH models ( $n=5-6$ per group): (i) feeding with a methionine choline-deficient (MCD) diet for 5 weeks and (ii) feeding with a high-fat (HF) diet containing 30\% lard, 1.25\% cholesterol and $0.5 \%$ sodium cholate for 12 weeks. ${ }^{24}$ Control animals were fed for 5 and 12 weeks, respectively, with standard chow. All diets were obtained from Sniff (Soest, Germany). All animals were 8 weeks of age at the start of the study and were maintained under specific pathogen-free and controlled conditions $\left(22^{\circ} \mathrm{C}\right.$, $55 \%$ humidity and $12 \mathrm{~h}$ day/night rhythm) and received human care in compliance with the guidelines outlined in the Guide for the Care and Use of Laboratory Animals. After the indicated time periods, animals were sacrificed and liver tissues were immediately snap-frozen and stored at $-80^{\circ} \mathrm{C}$ or were formalinfixed for subsequent analysis. Furthermore, $\mathrm{BMP}^{+/+}$and $\mathrm{BMP}^{-/-}$mice were subjected to a model of carbon tetrachloride $\left(\mathrm{CCl}_{4}\right)$-induced liver injury as described. ${ }^{25}$ In addition, we analysed liver tissues from wt mice that have been subjected to bile duct ligation (BDL) or sham operation. ${ }^{25} 26$

\section{(Immuno)histolochemical analysis}

For immunohistochemistry (IH) and HE staining, standard $5 \mu \mathrm{m}$ sections of formalin-fixed and paraffin-embedded tissue blocks were used. Immunohistochemical staining was performed using the following antibodies: anti-BMP6 (S-20) sc-27408 antibody (1:50; Santa Cruz Biotechnology) and anti-CD3 C7930 antibody (1:500; Sigma-Aldrich) as described. ${ }^{15} 27$ TUNEL assay was performed on paraffin sections as described. ${ }^{28}$ Prussian blue staining with $\mathrm{K}_{4} \mathrm{Fe}(\mathrm{CN})_{6} \cdot 3 \mathrm{H}_{2} \mathrm{O}$ and fast red counterstaining were performed based on standard procedures as described. ${ }^{15}$ Sirius red/fast green staining of formalin-fixed tissues was performed as described previously. ${ }^{29}$ Quantification of staining intensities was performed using image J software (National Institutes of Health; http://www.imagej.softonic.de). For analysis of the TMA, positivity for BMP6 or iron was defined as detectable cytoplasmatic staining, whereas cases designated as iron or BMP6 negative were devoid of staining.

\section{Western blot}

Protein extraction and western blot analysis were performed as described $^{27}$ applying antibodies against $\alpha$-smooth muscle actin ( $\alpha$-SMA) (ab5694 from Abcam, Cambridge, UK; 1:1000) or ß-actin (MAB1501 from Merck Millipore, Billerica, Massachusetts, USA; 1:500).

\section{Quantification of cytokine and chemokine levels}

TNF and MCP-1 levels in the supernatant of cells were measured by ELISA analysis using the TNF and MCP-1 DuoSet ELISA Development kits from R\&D Systems (Minneapolis, Minnesota, USA) according to the manufacturer's instructions. 
RNA isolation and quantitative real-time PCR (qRT-PCR) analysis

Isolation of total cellular RNA from cultured cells and liver tissues and reverse transcription were performed as described. ${ }^{15}$ Quantitative real-time PCR was done with specific sets of primers (see online supplementary table) applying LightCycler technology as described. ${ }^{4}$ All other mRNA expression analyses were performed using QuantiTect Primer Assays according to the manufacturer's instructions (Qiagen, Hilden, Germany). The amplification of cDNA derived from murine 18S RNA (for: 5' AAA CGG CTA CCA CAT CCA AG; rev: 5' CCT CCA ATG GAT CCT CGT TA) was used for normalisation.

\section{Quantification of hepatic iron and triglyceride content}

Hepatic lipids were extracted using the method of Bligh and Dyer with slight modifications. ${ }^{20}$ Hepatic triglyceride levels were quantified using the GPO-triglyceride kit (Sigma, Deisenhofen, Germany) as described. ${ }^{20}$ Hepatic iron concentration was determined by atomic absorption spectrophotometry as described. ${ }^{30}$

\section{Statistical analyses}

Results are expressed as mean $\pm S E$ (range) or per cent. Comparison between groups was made using the Student's unpaired $t$ test. A p value $<0.05$ was considered statistically significant. Contingency table analysis and the two-sided Fisher's exact test were used to study the statistical association between clinicopathological and immunohistochemical variables. All calculations were performed by using the GraphPad Prism Software (GraphPad Software, Inc., San Diego, USA) or SPSS (SPSS, Chicago, Illinois, USA).

\section{RESULTS}

\section{BMP6 expression in NAFLD}

First, we analysed hepatic BMP6 expression in different murine models of chronic liver injury. After 2 weeks of BDL as well as after 6 weeks of repeated carbon tetrachloride $\left(\mathrm{CCl}_{4}\right)$ application, BMP6 expression was not significantly altered compared with sham-treated mice (figure 1A). In contrast, after feeding a $\mathrm{NASH}$-inducing $\mathrm{HF}$ diet or a NASH-inducing MCD diet, BMP6 expression was significantly increased compared with mice fed with control chow (ctr.) (figure 1B). Also, in liver tissues of 12 patients with NAFLD, BMP6 mRNA expression was significantly increased while in 15 cirrhotic human liver tissues from patients with alcoholic liver disease and chronic viral hepatitis $\mathrm{B}$ or $\mathrm{C}$ infection BMP6 expression was similar to normal human liver tissues $(n=8)$ (figure $1 \mathrm{C})$.
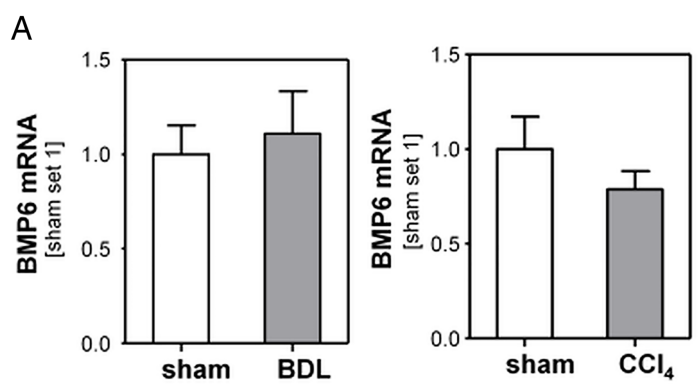

C

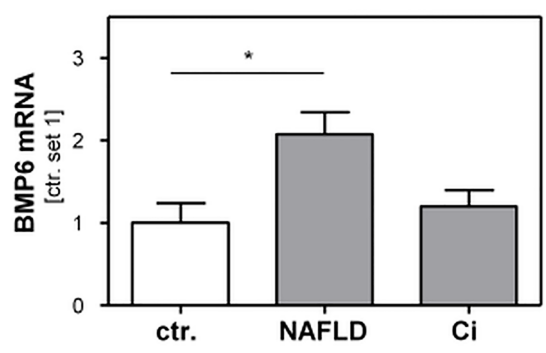

D
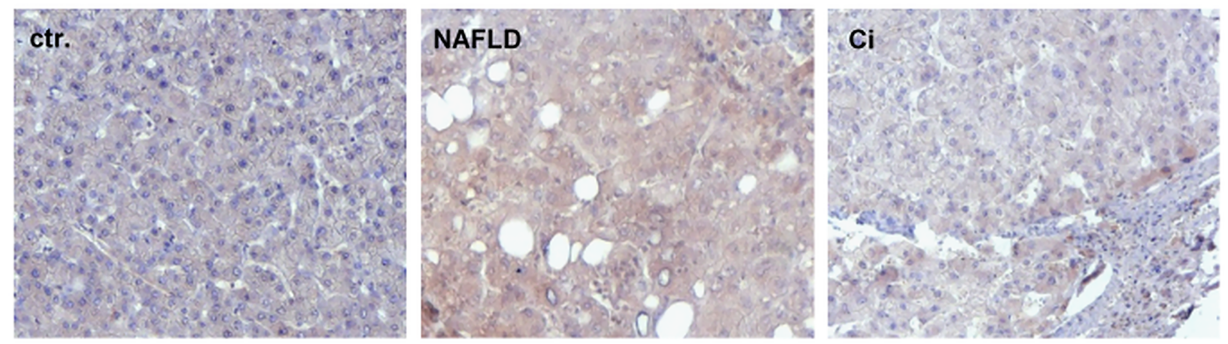

Figure 1 BMP6 expression in non-alcoholic fatty liver disease (NAFLD). (A) Hepatic mRNA expression of BMP6 in different murine models of chronic liver injury. (B) Hepatic mRNA expression of BMP6 in different murine models of NAFLD. (C) BMP6 mRNA expression in human liver specimens with different diagnostic findings. (D) Representative images of immunohistochemical staining for BMP6 of normal human liver tissue (ctr.) and liver tissues from NAFLD and cirrhotic (Ci) human livers. (E) BMP6 mRNA expression in hepatocytes in an in vitro model of cellular lipid accumulation ( $\left.{ }^{*} \mathrm{p}<0.05\right)$. 
Immunohistochemical analysis of BMP6 protein expression revealed hepatocytes as cellular source of hepatic BMP6 expression in human NAFLD livers with a distinct cytoplasmic staining pattern (figure 1D). Next, we applied IH and TMA technology to assess BMP6 expression in human liver tissues with different degree of steatosis and inflammation. ${ }^{21} \mathrm{IH}$ was informative in 110 cases. For descriptive data analysis, livers were separated into tissues with positive and negative BMP6 immunosignal (representative examples are depicted in online supplementary figure S1). BMP6 immunoreactivity significantly correlated with the degree of hepatic steatosis, while no association was found with hepatic inflammation, ballooning of hepatocytes or patients' age and sex (table 1). Also, in an in vitro model of cellular lipid accumulation, ${ }^{20}$ we observed a significant induction of BMP6 expression in primary human and murine hepatocytes (figure $1 \mathrm{E}$ and see online supplementary figure S2A). In contrast, stimulation with TNF- $\alpha$, a proinflammatory cytokine that plays a pivotal role in NAFLD development and progression, did not affect BMP6 expression in primary human hepatocytes $(\mathrm{PHH})$ (see online supplementary figure S2B). Fitting to this finding, there was no correlation between TNF- $\alpha$ and BMP6

Table 1 BMP6 immunoreactivity (IR) in liver tissues of 110 patients with NAFLD and controls in relation to clinicopathological characteristics

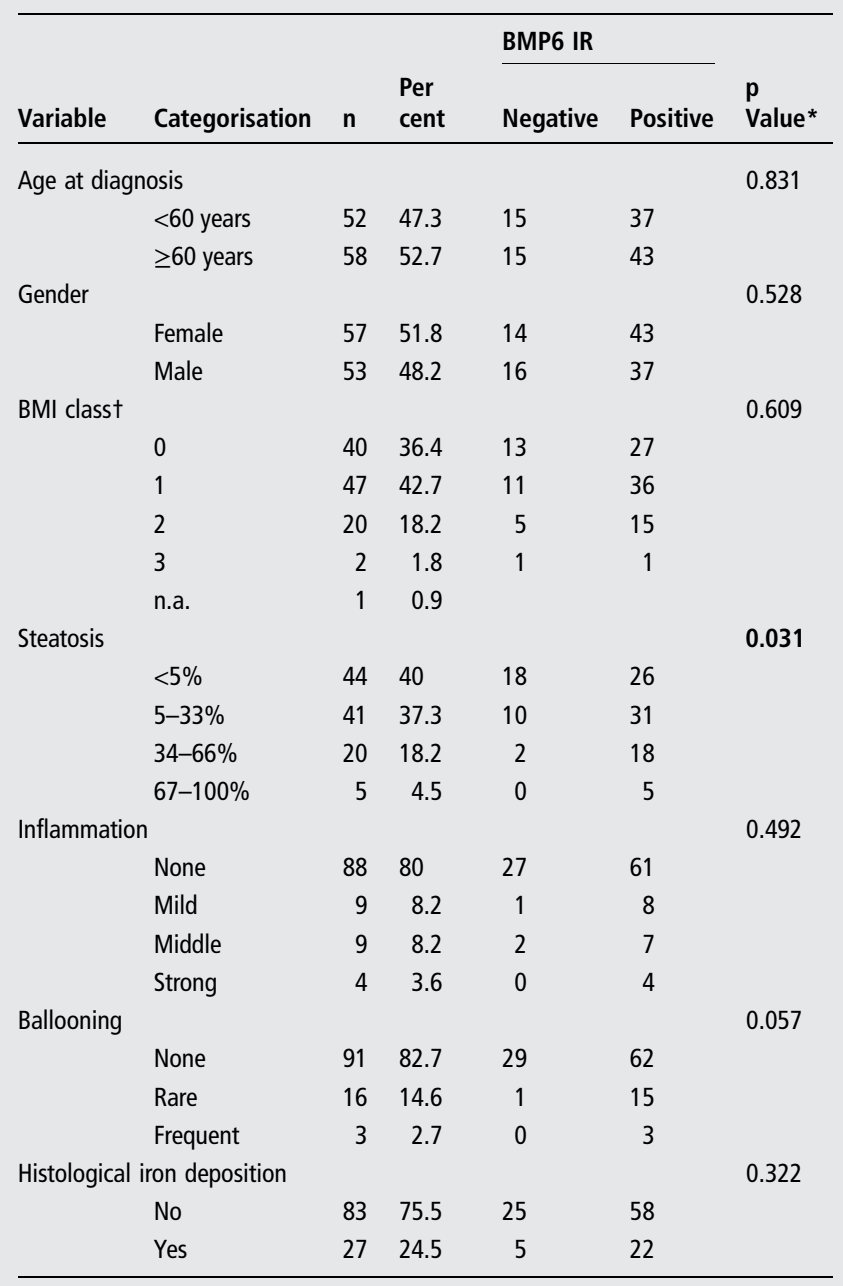

${ }^{*}$ Fisher's exact test (two-sided); bold value representing $\mathrm{p}$ value $<0.05$. tClass $0:<25 \mathrm{~kg} / \mathrm{m}^{2} ;$ class $1: \geq 25.0$ and $\leq 29.9 \mathrm{~kg} / \mathrm{m}^{2}$; class $2:>29.9$ and $\leq 34.9 \mathrm{~kg} / \mathrm{m}^{2}$; class $3 \geq 35.0 \mathrm{~kg} / \mathrm{m}^{2}$.

n.a., not available. expression in human NAFLD tissues (see online supplementary figure S2C). In addition to BMP6, we found also increased BMP2 and BMP4 in both NAFLD models (see online supplementary figure S3A-D) but not in the in vitro model of hepatocellular steatosis (see online supplementary figure S3E,F). As described in the introduction section, expression of BMP2 and BMP4 has been reported in acute liver injury. ${ }^{6}$ Furthermore, $\mathrm{BMP} 2$, but not BMP4 or BMP6, expression was found to be significantly elevated in a model of chronic alcohol feeding in mice. $^{31}$ Together, these data indicate that increased hepatic BMP6 expression in NAFLD results from hepatocellular lipid accumulation independent of liver inflammation and injury.

\section{Functional role of BMP6 in NAFLD}

To get insight into the function of enhanced BMP6 expression in NAFLD, we compared BMP6-deficient $\left(\mathrm{BMP}^{--}\right)$mice ${ }^{23}$ and wt mice in two different well-established dietary NASH models. ${ }^{32}$

First, we fed $\mathrm{BMP}^{-/-}$and wt mice with a MCD diet or control chow (ctr.) for 5 weeks. MCD-diet caused similar mainly macrovesicular hepatic steatosis in $\mathrm{BMP}^{-/-}$and wt mice (figure 2A), and also hepatic triglyceride levels were similar in MCD-fed $\mathrm{BMP}^{-/-}$and wt mice (figure 2B). Also, the number of apoptotic cells in the liver and serum transaminase levels did not significantly differ between $\mathrm{BMP}^{-/-}$and wt mice fed with the MCD diet (figure $2 \mathrm{C}$ and see online supplementary figure $\mathrm{S} 4 \mathrm{~A})$. However, BMP6 $6^{-/-}$mice showed higher hepatic expression of proinflammatory cytokines and chemokines (figure 2D and see online supplementary figure S4B-D) and immune cell infiltration (figure 2E) in response to MCD-feeding compared with wt mice. Furthermore, $\alpha$-SMA, a well-established marker of HSC activation, was significantly higher expressed in the liver of MCD-fed $\mathrm{BMP}^{-/-}$mice compared with MCD-fed wt mice (figure $2 \mathrm{~F}$ ). The activation of HSCs is one of the central pathophysiological mechanisms of liver fibrogenesis. ${ }^{33}$ Fitting to this, also hepatic expression of collagen $\alpha \mathrm{I}(1)$ (Coll-I), the most abundant extracellular matrix (ECM) protein of fibrotic liver tissue, and TGF-ß1, a strong profibrogenic cytokine, was significantly higher in $\mathrm{MCD}$-fed $\mathrm{BMP}^{-/-}$mice compared with MCD-fed wt mice (figure $2 \mathrm{G}$ and see online supplementary figure S4E). In line with this, sirius red/fast green staining revealed pronounced ECM deposition in the liver of MCD-fed $\mathrm{BMP}^{-/-}$mice compared with wt mice (figure $2 \mathrm{H}$ ).

As second model we applied a HF (30\% lard) diet supplemented with cholesterol and cholate, which has been shown to induce pathological changes closely resembling human NASH. ${ }^{21}{ }^{24}$ Feeding this HF diet for 12 weeks caused similar hepatocellular ballooning and mainly microvesicular steatosis in liver of $\mathrm{BMP}^{-/-}$and wt mice (figure $3 \mathrm{~A}$ ). Also, hepatic triglyceride levels, number of apoptotic cells in the liver and serum transaminases were similar in HF diet-fed $\mathrm{BMP}^{-1-}$ and wt mice (figure 3B, C and see online supplementary figure S5A). In contrast, proinflammatory gene expression and immune cell infiltration in response to feeding the HF diet was significantly higher in livers of $\mathrm{BMP}^{-/-}$compared with wt mice (figure $3 \mathrm{D}, \mathrm{E}$ and see online supplementary figure S5B-D). Within the feeding time of 12 weeks, the HF diet caused a slight induction of hepatic TGF-ß1 and Coll- $\alpha \mathrm{I}(1)$ mRNA expression in wt mice (see online supplementary figure S5E and figure 3G), and histological analysis did not show significant ECM deposition in wt mice (figure $3 \mathrm{H}$ ). In contrast, the $\mathrm{HF}$ diet induced a marked increase of hepatic $\alpha$-SMA expression as well as evident hepatic fibrosis in $\mathrm{BMP}^{-/-}$mice (figure $3 \mathrm{~F}-\mathrm{H}$ ). 
A
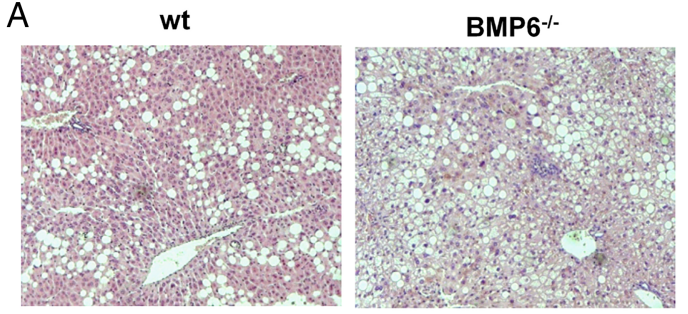

C

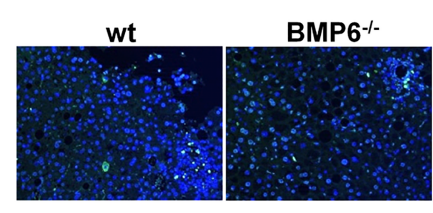

$E$

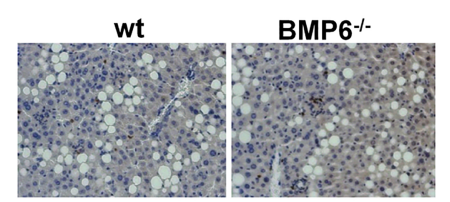

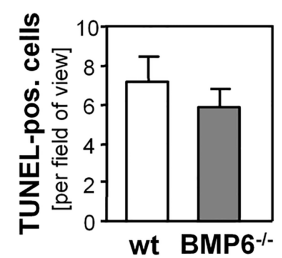

wt $\mathrm{BMP6}^{-1}$

$\mathrm{D}$

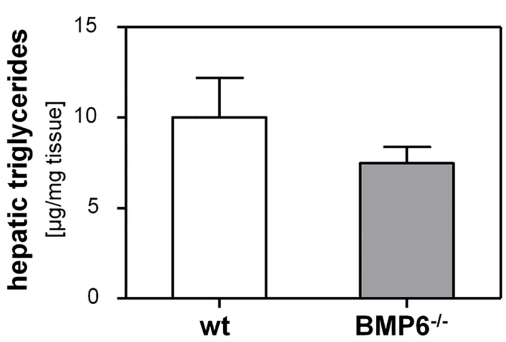

$\mathrm{F}$
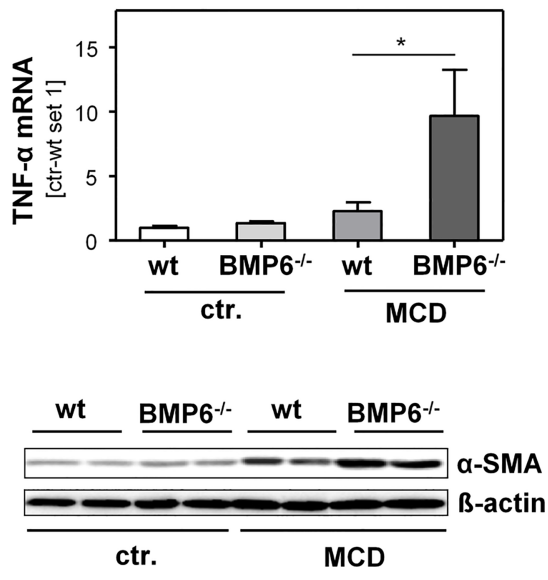

G

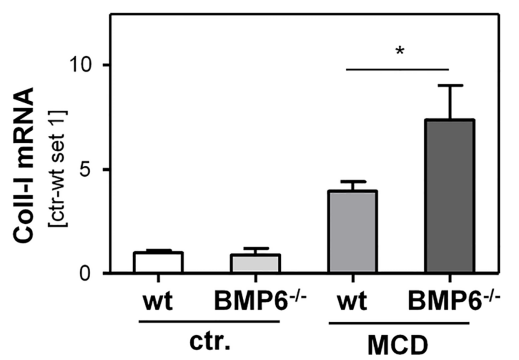

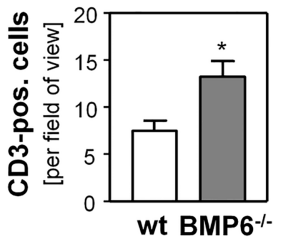

$\mathrm{H}$
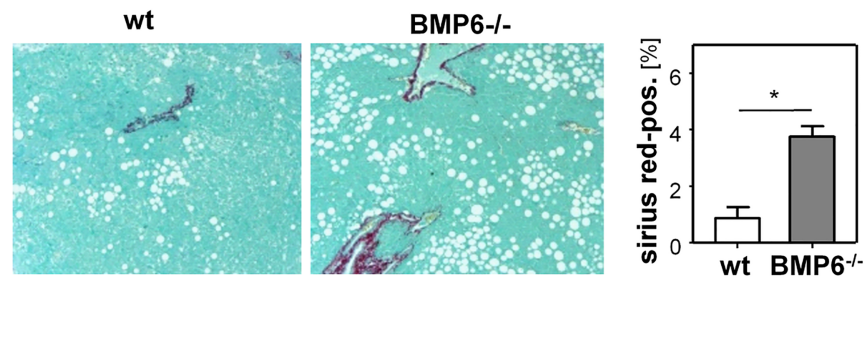

Figure 2 Effect of BMP6 deficiency in mice fed with a non-alcoholic steatohepatitis-inducing methionine choline-deficient (MCD) diet. (A) Hematoxylin/ eosin (H/E) staining of liver sections from wild-type mice (wt) and BMP6-deficient $\left(\mathrm{BMPG}^{-l-}\right)$ mice after feeding a MCD diet for 5 weeks. (B) Hepatic triglyceride levels in MCD-fed $\mathrm{BMP6}^{-l-}$ and wt mice. (C) Representative images of TUNEL staining of hepatic tissue from MCD-fed BMP6 ${ }^{-l-}$ and wt mice (left panel). Numbers of TUNEL positive cells were counted in three different fields per mouse (right panel). (D) Hepatic mRNA expression of tumour necrosis factor- $\alpha$ (TNF- $\alpha$ ) in response to MCD feeding compared with control animals (ctr.) fed with standard chow. (E) Analysis of hepatic immune cell infiltration by CD3 immunohistochemical staining of livers of MCD-fed wt mice and $\mathrm{BMP6}^{-1-}$ mice (left panel). Numbers of CD3 positive cells were counted in three different fields per mouse (right panel). (F) Analysis of hepatic $\alpha$-smooth muscle actin ( $\alpha$-SMA) protein expression by western blot technique of liver cell lysates of control-fed and MCD-fed BMP6 ${ }^{--}$and wt mice. $\beta$-Actin was used as loading control. (G) Hepatic expression of collagen alpha I(1) (Coll-I) in response to MCD feeding compared with control animals. (H) Representative images of sirius red/fast green staining of liver sections from wt and $\mathrm{BMP6}^{-l-}$ mice after feeding MCD diet (left panel). Quantification of sirius red-positive areas using image analysis (right panel) $\left({ }^{*} \mathrm{p}<0.05\right)$.

In summary, these results suggest that BMP6 protects against hepatic inflammation and fibrosis in mice fed with a NASH-inducing diet.

To analyse whether this effect is only related to NAFLD induced injury, we also applied the model of toxic liver injury by $\mathrm{CCl}_{4}{ }^{25}$ to $\mathrm{BMP}^{-/-}$and wt mice. Also in this model, hepatic expression of proinflammatory and profibrogenic genes was higher in $\mathrm{BMP6}^{-/-}$mice compared with wt mice (see online supplementary figure S6). Although only differences in MCP-1 and $\alpha$-SMA expression reached the level of significance, these data suggest that also in this model, BMP6 expression exhibits anti-inflammatory and antifibrogenic effects. Still, together with the missing induction of BMP6 upon liver injury not related to NAFLD (figure 1), the observed mild difference between $\mathrm{BMP6}^{-1-}$ and wt mice in the $\mathrm{CCl}_{4}$ model suggests that the hepatoprotective effect of the BMP6 pathway mainly plays a role in fatty liver disease.

\section{Mechanisms underlying the hepatoprotective effect of BMP6 in NAFLD}

In search for the mechanisms by which BMP6 exhibits antiinflammatory and antifibrogenic effects in NAFLD, we first looked at hepatic iron deposition since BMP6 is a critical regulator of hepcidin expression, ${ }^{13}{ }^{14}$ and increasing data from experimental and clinical studies indicate that iron sustains disease activity and/or contributes to its progression in NAFLD. ${ }^{35}$ The MCD model is known to enhance hepatic iron uptake, ${ }^{36}$ and as shown in our previous study ${ }^{15}$ hepatic hepcidin expression was significantly lower (figure 4A) while hepatocellular iron deposition was markedly increased in BMP6 $6^{-/-}$mice compared with 
A

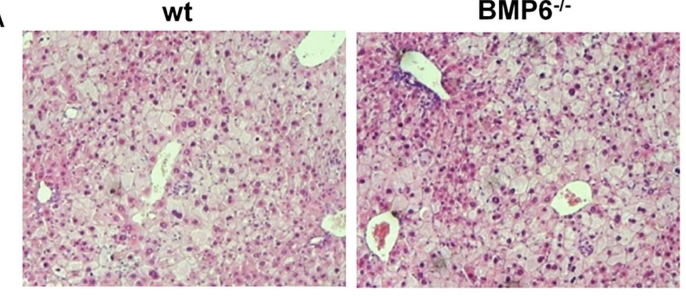

C
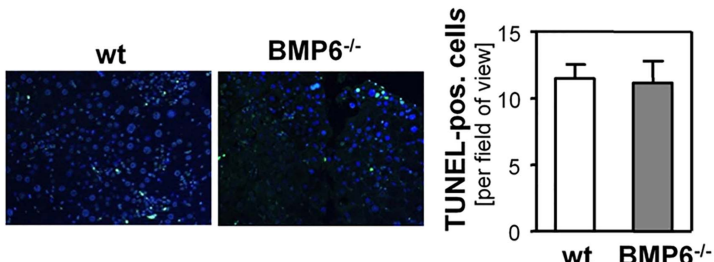

E

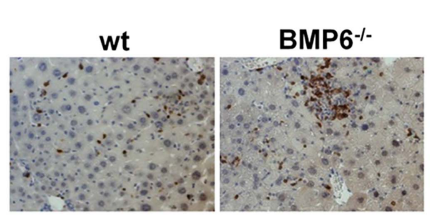

G

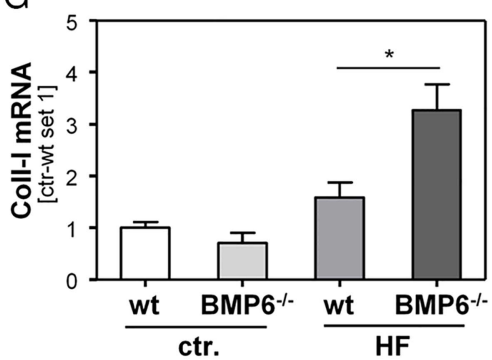

B

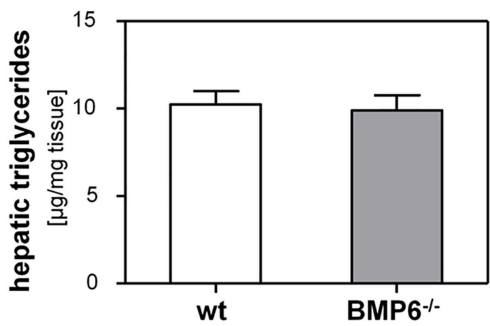

D
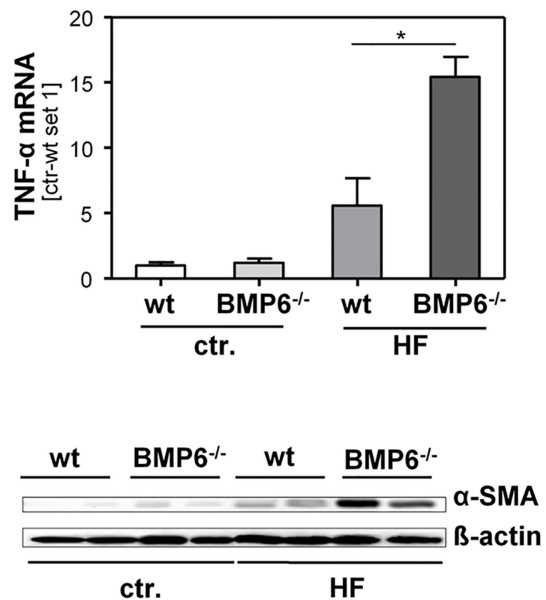

$\mathrm{BMP6}^{-1}$

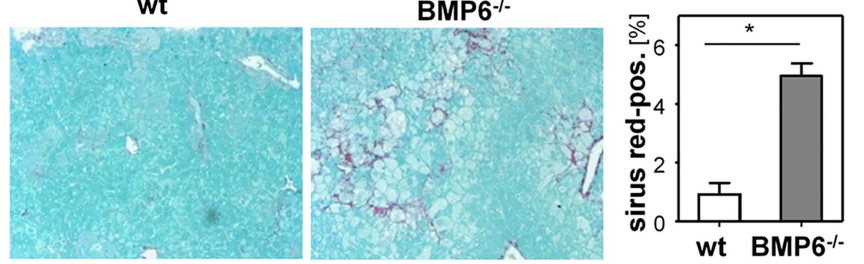

Figure 3 Effect of BMP6 deficiency in mice fed with a non-alcoholic steatohepatitis-inducing high-fat (HF) diet. (A) HE staining of liver sections from wt mice (wt) and BMP6-deficient (BMP6 ${ }^{-l-}$ ) mice after feeding a HF diet for 12 weeks. (B) Hepatic triglyceride levels in HF-fed BMP6 ${ }^{-l-}$ and wt mice. (C) Representative images of TUNEL staining of hepatic tissue from HF diet-fed BMP6 ${ }^{-1-}$ and wt mice (left panel). Numbers of TUNEL positive cells were counted in three different fields per mouse (right panel). (D) Hepatic mRNA expression of tumour necrosis factor- $\alpha$ (TNF- $\alpha$ ) in response to HF feeding compared with control animals (ctr.) fed with standard chow. (E) Analysis of hepatic immune cell infiltration by CD3 immunohistochemical staining of livers of $\mathrm{HF}$ diet-fed wt mice and $\mathrm{BMP6}^{-l-}$ mice (left panel). Numbers of $\mathrm{CD} 3$ positive cells were counted in three different fields per mouse (right panel). (F) Analysis of hepatic $\alpha$-smooth muscle actin ( $\alpha$-SMA) protein expression by western blot technique of liver cell lysates of control-fed and HF diet-fed BMPG ${ }^{-1-}$ and wt mice. $\beta$-Actin was used as loading control. (G) Hepatic expression of collagen alpha I(1) (Coll-I) in response to HF diet feeding compared with control animals. (H) Representative images of sirius red/fast green staining of liver sections from wt and $\mathrm{BMPG}^{-1-}$ mice after feeding HF diet (left panel). Quantification of sirius red-positive areas using image analysis (right panel) $\left({ }^{*} \mathrm{p}<0.05\right)$.

wt control mice in response to MCD-diet feeding (figure 4C, D). In contrast, hepatic hepcidin expression was only slightly lower in $\mathrm{BMP}^{-/-}$mice compared with wt control mice fed with the NASH-inducing HF diet (figure 4B) and this diet also did not lead to significant iron accumulation in $\mathrm{BMP}^{-/-}$mice compared with wt control mice (figure 4C, D). These data indicate that at least in the HF diet model disruption of iron metabolism is not the cause for increased hepatic injury in $\mathrm{BMP}^{-/-}$mice. Moreover, these findings further underscore that the increased BMP6 expression observed in wt livers after feeding the MCD or HF diets is caused by hepatic steatosis and is not or only partially caused by increased iron deposition. In line with this, Prussian blue staining of the human NAFLD-TMA revealed more often visible iron deposition in NAFLD $(20 / 65 ; 30.8 \%)$ compared with non-steatotic liver tissues $(7 / 45 ; 15.6 \% ; \mathrm{p}=0.07)$ (see online supplementary figure
S7A). Representative examples of iron-positive and iron-negative liver tissues are depicted in online supplementary figure S7B. However, there was no correlation between iron deposition and immunohistochemical BMP6 detection (table 1). Moreover, feeding with NASH-inducing diets did not lead to significantly different hepatic expression levels of other BMPs, such as BMP2 or BMP4, which might be responsible for the observed differences between $\mathrm{BMP}^{-/-}$and wt control mice (see online supplementary figure $\mathrm{S} 8$ ).

In further search for the BMP6 mechanisms of hepatic protection, we assessed the effects of BMP6 on Kupffer cells, the liver-resident macrophages. Kupffer cells initiate and perpetuate the inflammatory response by releasing inflammatory mediators that contribute to inflammatory cell recruitment and development of fibrosis in NAFLD. ${ }^{37}$ However, stimulation with recombinant BMP6 did not significantly affect MCP-1 and TNF- $\alpha$ 

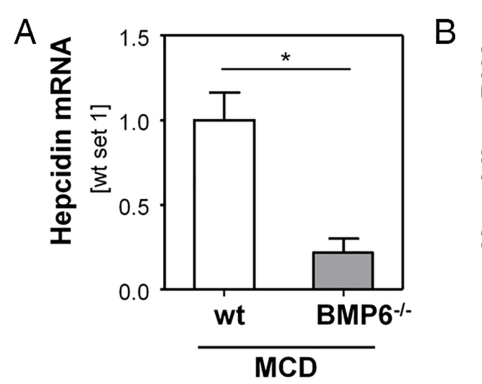
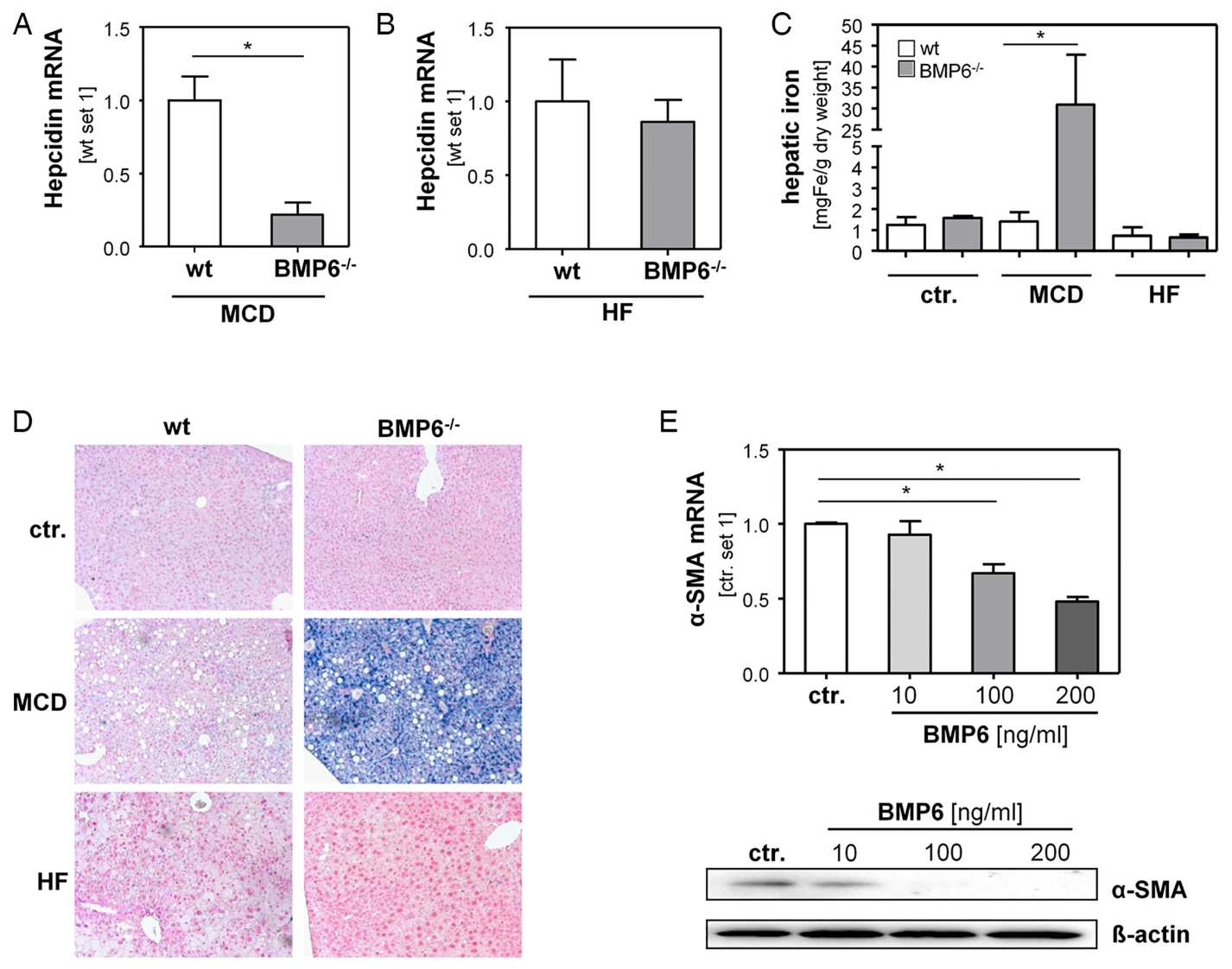

$\mathrm{E}$

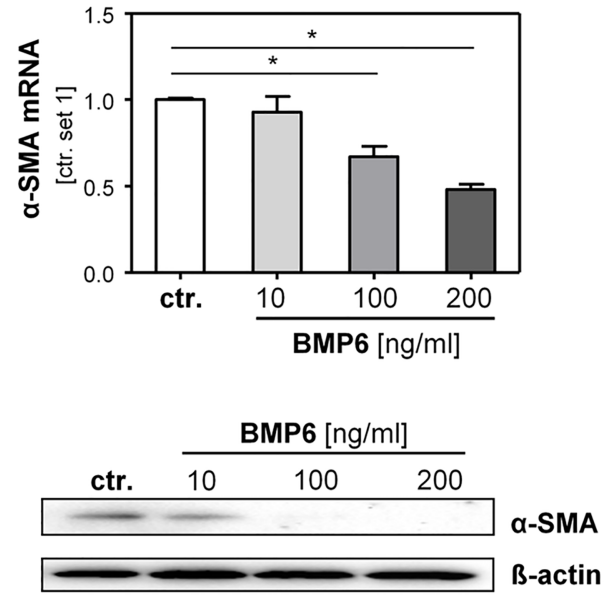

$\mathrm{F}$
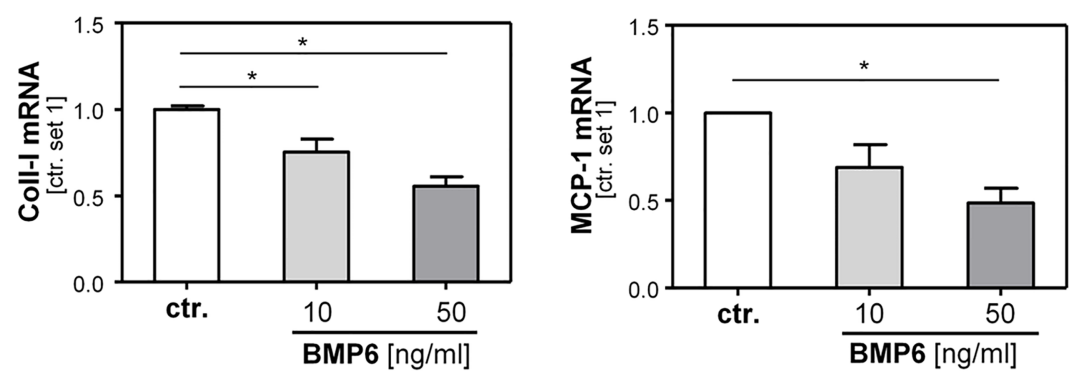

G

$\mathrm{H}$
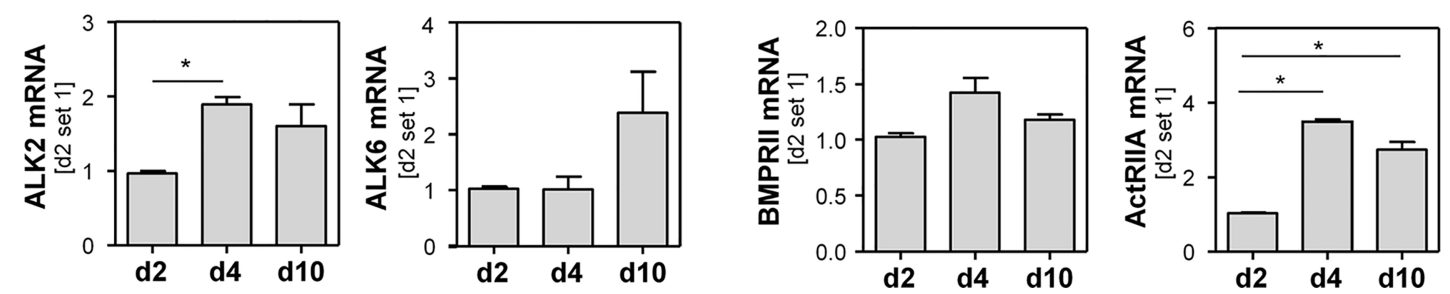

Figure 4 Mechanisms underlying the hepatoprotective effect of BMP6 in non-alcoholic fatty liver disease. (A) Hepatic hepcidin expression in methionine choline-deficient (MCD) diet-fed and (B) high-fat (HF) diet-fed BMP6 ${ }^{-I-}$ and wt mice. (C) Hepatic iron levels in control-fed, MCD diet-fed and HF diet-fed BMP6 ${ }^{-I-}$ and wt mice. (D) Prussian blue staining of liver sections from control-fed, MCD diet-fed and HF diet-fed BMP6 ${ }^{-I-}$ and wt mice. (E) Expression of $\alpha$-smooth muscle actin ( $\alpha$-SMA) mRNA (upper panel) and protein (lower panel) in activated primary murine hepatic stellate cells (HSCs) after exposure to BMP6 in doses as indicated. (F) mRNA expression of collagen alpha I(1) (Coll-I) and MCP-1 in activated murine HSCs after BMP6 stimulation. (G) Analysis of mRNA expression of type I and (H) type II BMP-receptors in human HSCs in vitro at different time points after isolation (day 2, day 4 , and day 10$)\left({ }^{*} p<0.05\right)$.

secretion of primary murine Kupffer cells in vitro (see online supplementary figure S9). This suggests that Kupffer cells are not the primary target cells by which BMP6 exerts its diseasemodulating and fibrosis-modulating effects.
Next, we analysed the effects of BMP6 on HSCs. First, we aimed to analyse the effect of BMP6 on the in vitro activation process of HSCs. Two days after isolation, primary human and murine HSCs were exposed to different BMP6 doses for 3 days. 
Quantitative RT-PCR analysis revealed that BMP6 stimulation dose-dependently reduced the expression $\alpha$-SMA in murine and human HSCs (figure 4E and see online supplementary figure S10A). In contrast, in vitro activation of HSCs isolated from $\mathrm{BMP6}^{-/-}$and wt control mice did not show significant differences in $\alpha$-SMA and Coll-I expression (see online supplementary figure S10B). Once they are activated, HSCs are characterised by excessive expression of profibrogenic genes and ECM proteins. Furthermore, activated HSCs significantly contribute to the initiation and perpetuation of hepatic inflammation by (de novo) expression of proinflammatory cytokines and chemokines. ${ }^{38}$ Noteworthy, BMP6 stimulation of activated HSCs led to a reduced expression of Coll-I as well as the chemokine monocyte chemotactic protein-1 (MCP-1) in activated murine and human HSCs (figure 4F and see online supplementary figure S10C). Together, these results indicate that BMP6 inhibits the activation process of HSCs and proinflammatory and profibrogenic gene expression of already activated HSCs. Fitting to this was the expression pattern of type I (Alk2, Alk3, Alk6) and type II (BMPRII, ActRIIA) BMP receptors known to mediate BMP6 signal transduction ${ }^{39}$ during the in vitro activation course of human HSCs. ALK2, ALK6, BMPRII and ActRIIA expression was already detectable in quiescent HSCs (d2), and ALK2, ALK6 and ActRIIA further increased during HSC activation (d4, d10) (figure 4G, H). In contrast, no ALK3 expression was detectable in quiescent or activated HSCs (data not shown), which is in line with a previous study reporting that this type I receptor is not expressed in the liver. ${ }^{40}$

\section{DISCUSSION}

The first aim of this study was to investigate the expression of BMP6 in chronic liver diseases. Analysis of hepatic BMP6 expression in experimental murine models and patient samples revealed an increase restricted to NAFLD but not in other chronic liver diseases. NAFLD is characterised by increased uptake and accumulation of lipids in hepatocytes. Simple hepatic steatosis may progress to non-alcoholic steatohepatitis (NASH) with hepatic inflammation, hepatocellular injury and fibrosis. ${ }^{41}$ Our in vitro and in vivo data strongly indicated that the increased BMP6 expression in NAFLD was not related to hepatocellular injury or inflammation but was caused by hepatocellular lipid accumulation. Previous studies demonstrated that also chronic iron overload can lead to increased hepatic BMP6 levels. ${ }^{42}$ Furthermore, several studies suggest that iron homeostasis is disturbed in NAFLD, ${ }^{43} 44$ and although conflicting results have been obtained, most studies support the hypothesis that iron plays a role in hepatic lipogenesis. ${ }^{45}$ Still, systemic and hepatic iron levels are increased also in other types of liver diseases but NAFLD, and we did not find a correlation between BMP6 expression and histological evident iron deposition in human NAFLD tissues. Together, these findings indicate that iron is not the critical factor causing enhanced BMP6 levels in fatty livers. Further studies have to identify the underlying mechanism of this phenomenon.

In the present study, we further aimed to determine the function of enhanced BMP6 expression in NAFLD. We observed that genetic loss of BMP6 aggravated hepatic inflammation, and even more, the development of hepatic fibrosis in experimental NAFLD models. In vitro analysis revealed a significant inhibitory effect of BMP6 on the activation of HSCs as well as proinflammatory and profibrogenic gene expression of already activated HSCs. These findings suggest that the observed protective effect of BMP6 in murine NAFLD models was at least in part mediated via direct effects on HSCs. An antifibrotic effect of BMP6 has also been described in a model of chronic renal injury. ${ }^{46}$
Different than in NAFLD, BMP6 was downregulated in diseased kidneys of wt mice. Furthermore, BMP6-deficient mice showed a significant increase of iron deposition in the kidney upon injury. ${ }^{46}$ In our study, only in one model (ie, the MCD diet), which is known to enhance hepatic iron uptake, ${ }^{36}$ hepatocellular iron deposition was markedly increased in $\mathrm{BMP}^{-/-}$mice compared with wt control mice. Furthermore, we did not find a significant correlation between histological iron deposition and BMP6 expression in human NAFLD patients. These data suggest that hepatic iron accumulation, which is considered a putative element that interacts with oxygen radicals in inducing liver damage and fibrosis, ${ }^{47}$ is not the main cause of enhanced hepatic injury of BMP6-deficient mice in NAFLD models. Still, under certain circumstances, defects of BMP6 signalling may further aggravate NAFLD progression via effects on iron homeostasis. Thus, one may speculate whether the role of BMP6 becomes even more critical with aging. Also, in the present study, we may have seen more pronounced effects of BMP6-deficiency in older mice since $\mathrm{BMP}^{-/-}$mice develop progressive hepatic iron deposition with aging. ${ }^{15}$ However, young $\mathrm{BMP}^{-/-}$mice show no overt defects in the liver. ${ }^{23}$ It appears that BMP6 plays a minor role in hepatic development, or that its functions are covered in this context by functional redundancy from other BMPs. In contrast, compensatory mechanisms are apparently not sufficient to protect the adult animals during hepatic injury in NAFLD. This is notable as, for example, BMP6 and BMP7 share $87 \%$ amino acid identity and signal via the type I receptors activin-like kinase ALK2, ALK3 and ALK6, ${ }^{48} 49$ and activated HSCs express high levels of these three, and other BMP receptors and coreceptors (BMPRII, ActRIIA and Eng). ${ }^{1050} 51$

The restricted increase of BMP6 in NAFLD but not in other liver diseases and the mild differences between $\mathrm{BMP}^{-/-}$and $\mathrm{wt}$ mice in the toxic $\mathrm{CCl}_{4}$ model suggest the BMP6 pathway mainly plays a role in NAFLD and that therapeutic efforts should be mainly directed at this entity. Future studies have to unravel whether the antifibrogenic effect of BMP6 is restricted to NAFLD and to further investigate the therapeutic potential of BMP6 in (other) models of chronic liver injury. Certainly, caution will be necessary not only in the light of BMP6's role in iron homeostasis. However, more detailed insight into BMP6 signalling in HSCs may allow the development of a tailored antifibrogenic therapeutic strategy for NAFLD and eventually also other chronic liver diseases.

Acknowledgements We are indebted to Martina Waeber, Birgitta Ott-Rötzer and Rudolf Jung for technical assistance, and Daniel Wagner for expert animal care. Furthermore, we thank Elizabeth Robertson (University of Oxford, England, UK) for kindly providing the BMP6-deficient mice and Florian Weber for pathological judgment of the human fibrotic tissue sections. We acknowledge the Human Tissue and Cell Research (HTCR) Foundation for supporting our research by making human liver tissue available.

Contributors SA, EW, CD, AK and MS performed the experiments and acquired the data. AH performed histological analysis. SA, AKB and $\mathrm{CH}$ analysed and interpreted the data. WET provided material support. CH and AKB designed and supervised the study. SA, CH and AKB drafted the manuscript, and all authors critically revised the manuscript.

Funding This work was supported by grants from the German Research Association (DFG) to $\mathrm{CH}$ and $\mathrm{AKB}$.

\section{Competing interests None.}

Ethics approval State controlled foundation HTCR and the local ethics committee of the University Regensburg.

Provenance and peer review Not commissioned; externally peer reviewed.

Data sharing statement There are no relevant data, which are not included in the manuscript. All available data forming the basis of this manuscript will be shared. 


\section{REFERENCES}

1 Bragdon B, Moseychuk O, Saldanha S, et al. Bone morphogenetic proteins: a critical review. Cell Signal 2011;23:609-20.

2 Singh A, Morris RJ. The Yin and Yang of bone morphogenetic proteins in cancer. Cytokine Growth Factor Rev 2010;21:299-313.

3 Maegdefrau U, Bosserhoff AK. BMP activated Smad signaling strongly promotes migration and invasion of hepatocellular carcinoma cells. Exp Mol Pathol 2012;92:74-81.

4 Maegdefrau U, Amann T, Winklmeier A, et al. Bone morphogenetic protein 4 is induced in hepatocellular carcinoma by hypoxia and promotes tumour progression. J Pathol 2009;218:520-9.

5 Li Q, Gu X, Weng H, et al. Bone morphogenetic protein-9 induces epithelial to mesenchymal transition in hepatocellular carcinoma cells. Cancer $\mathrm{Sci}$ 2013;104:398-408.

6 Nakatsuka R, Taniguchi M, Hirata M, et al. Transient expression of bone morphogenic protein-2 in acute liver injury by carbon tetrachloride. J Biochem 2007;141:113-19.

7 Oumi N, Taniguchi KA, Kanai AM, et al. A crucial role of bone morphogenetic protein signaling in the wound healing response in acute liver injury induced by carbon tetrachloride. Int J Hepatol 2012;2012:476820.

8 Herrera B, Sanchez A, Fabregat I. BMPS and liver: more questions than answers. Curr Pharm Des 2012;18:4114-25.

9 Chayanupatkul M, Honsawek S, Vejchapipat $P$, et al. Elevated serum bone morphogenetic protein 7 levels and clinical outcome in children with biliary atresia. Eur J Pediatr Surg 2009;19:246-50.

10 Tacke F, Gabele E, Bataille F, et al. Bone morphogenetic protein 7 is elevated in patients with chronic liver disease and exerts fibrogenic effects on human hepatic stellate cells. Dig Dis Sci 2007;52:3404-15.

11 Kinoshita K, limuro Y, Otogawa K, et al. Adenovirus-mediated expression of BMP-7 suppresses the development of liver fibrosis in rats. Gut 2007;56:706-14.

12 Zeisberg M, Yang C, Martino M, et al. Fibroblasts derive from hepatocytes in liver fibrosis via epithelial to mesenchymal transition. J Biol Chem 2007;282:23337-47.

13 Andriopoulos B Jr, Corradini E, Xia Y, et al. BMP6 is a key endogenous regulator of hepcidin expression and iron metabolism. Nat Genet 2009;41:482-7.

14 Meynard D, Kautz L, Darnaud V, et al. Lack of the bone morphogenetic protein BMP6 induces massive iron overload. Nat Genet 2009;41:478-81.

15 Arndt S, Maegdefrau U, Dorn C, et al. Iron-induced expression of bone morphogenic protein 6 in intestinal cells is the main regulator of hepatic hepcidin expression in vivo. Gastroenterology 2010;138:372-82.

16 Xia Y, Babitt JL, Sidis Y, et al. Hemojuvelin regulates hepcidin expression via a selective subset of BMP ligands and receptors independently of neogenin. Blood 2008;111:5195-204.

17 Babitt JL, Huang FW, Xia Y, et al. Modulation of bone morphogenetic protein signaling in vivo regulates systemic iron balance. J Clin Invest 2007;117:1933-9.

18 Muhlbauer M, Fleck M, Schutz C, et al. PD-L1 is induced in hepatocytes by viral infection and by interferon-alpha and -gamma and mediates $T$ cell apoptosis. J Hepatol 2006;45:520-8.

19 Weiss TS, Pahernik S, Scheruebl I, et al. Cellular damage to human hepatocytes through repeated application of 5-aminolevulinic acid. J Hepatol 2003;38:476-82.

20 Wobser H, Dorn C, Weiss TS, et al. Lipid accumulation in hepatocytes induces fibrogenic activation of hepatic stellate cells. Cell Res 2009;19:996-1005.

21 Dorn C, Riener MO, Kirovski G, et al. Expression of fatty acid synthase in nonalcoholic fatty liver disease. Int J Clin Exp Pathol 2010;3:505-14.

22 Kleiner DE, Brunt EM, Van Natta $\mathrm{M}$, et al. Design and validation of a histological scoring system for nonalcoholic fatty liver disease. Hepatology 2005;41:1313-21.

23 Solloway MJ, Dudley AT, Bikoff EK, et al. Mice lacking Bmp6 function. Dev Genet 1998;22:321-39.

24 Matsuzawa N, Takamura T, Kurita $\mathrm{S}$, et al. Lipid-induced oxidative stress causes steatohepatitis in mice fed an atherogenic diet. Hepatology 2007;46:1392-403.

$25 \mathrm{Xu}$ W, Hellerbrand C, Kohler UA, et al. The Nrf2 transcription factor protects from toxin-induced liver injury and fibrosis. Lab Invest 2008;88:1068-78.

26 Gabele E, Froh M, Arteel GE, et al. TNFalpha is required for cholestasis-induced liver fibrosis in the mouse. Biochem Biophys Res Commun 2009;378:348-53.
27 Dorn C, Engelmann JC, Saugspier M, et al. Increased expression of c-Jun in nonalcoholic fatty liver disease. Lab Invest 2014;94:394-408.

28 Braig S, Bosserhoff AK. Death inducer-obliterator 1 (Dido1) is a BMP target gene and promotes BMP-induced melanoma progression. Oncogene 2013;32:837-48.

29 Bergheim I, Guo L, Davis MA, et al. Critical role of plasminogen activator inhibitor-1 in cholestatic liver injury and fibrosis. J Pharmacol Exp Ther 2006;316:592-600.

30 Saugspier M, Dorn C, Thasler WE, et al. Hop bitter acids exhibit anti-fibrogenic effects on hepatic stellate cells in vitro. Exp Mol Pathol 2012;92:222-8.

31 Gerjevic LN, Liu N, Lu S, et al. Alcohol Activates TGF-Beta but Inhibits BMP Receptor-Mediated Smad Signaling and Smad4 Binding to Hepcidin Promoter in the Liver. Int J Hepatol 2012;2012:459278.

32 Liu Y, Meyer C, Xu C, et al. Animal models of chronic liver diseases. Am J Physiol Gastrointest Liver Physiol 2013;304:G449-68.

33 Henderson NC, Arnold TD, Katamura Y, et al. Targeting of alphav integrin identifies a core molecular pathway that regulates fibrosis in several organs. Nat Med 2013;19:1617-24.

34 Mederacke I, Hsu CC, Troeger JS, et al. Fate tracing reveals hepatic stellate cells as dominant contributors to liver fibrosis independent of its aetiology. Nat Commun 2013;4:2823.

35 Corradini E, Pietrangelo A. Iron and steatohepatitis. J Gastroenterol Hepatol 2012;27(Suppl 2):42-6.

36 Kirsch $\mathrm{R}$, Sijtsema HP, Tlali $\mathrm{M}$, et al. Effects of iron overload in a rat nutritional model of non-alcoholic fatty liver disease. Liver Int 2006;26:1258-67.

37 Marra F, Lotersztajn S. Pathophysiology of NASH: perspectives for a targeted treatment. Curr Pharm Des 2013;19:5250-69.

38 Dorn C, Heilmann J, Hellerbrand C. Protective effect of xanthohumol on toxin-induced liver inflammation and fibrosis. Int J Clin Exp Pathol 2012;5:29-36.

39 Mazerbourg S, Hsueh AJ. Genomic analyses facilitate identification of receptors and signalling pathways for growth differentiation factor 9 and related orphan bone morphogenetic protein/growth differentiation factor ligands. Hum Reprod Update 2006;12:373-83.

40 ten Dijke $\mathrm{P}$, Ichijo $\mathrm{H}$, Franzen $\mathrm{P}$, et al. Activin receptor-like kinases: a novel subclass of cell-surface receptors with predicted serine/threonine kinase activity. Oncogene 1993;8:2879-87.

41 Cohen JC, Horton JD, Hobbs HH. Human fatty liver disease: old questions and new insights. Science 2011;332:1519-23.

42 Corradini E, Meynard D, Wu Q, et al. Serum and liver iron differently regulate the bone morphogenetic protein 6 (BMP6)-SMAD signaling pathway in mice. Hepatology 2011;54:273-84.

43 Datz C, Felder TK, Niederseer D, et al. Iron homeostasis in the metabolic syndrome. Eur J Clin Invest 2013:43:215-24.

44 Wallace DF, Subramaniam VN. Co-factors in liver disease: the role of HFE-related hereditary hemochromatosis and iron. Biochim Biophys Acta 2009;1790:663-70.

45 Ahmed U, Latham PS, Oates PS. Interactions between hepatic iron and lipid metabolism with possible relevance to steatohepatitis. World J Gastroenterol 2012:18:4651-8.

46 Dendooven A, van Oostrom 0, van der Giezen DM, et al. Loss of endogenous bone morphogenetic protein-6 aggravates renal fibrosis. Am J Pathol 2011;178:1069-79.

47 Fujita N, Takei Y. Iron overload in nonalcoholic steatohepatitis. Adv Clin Chem 2011;55:105-32

48 Ebisawa T, Tada K, Kitajima I, et al. Characterization of bone morphogenetic protein-6 signaling pathways in osteoblast differentiation. J Cell Sci 1999;112(Pt 20):3519-27.

49 ten Dijke P, Yamashita H, Sampath TK, et al. Identification of type I receptors for osteogenic protein-1 and bone morphogenetic protein-4. J Biol Chem 1994;269:16985-8.

50 Meurer SK, Tihaa L, Borkham-Kamphorst E, et al. Expression and functional analysis of endoglin in isolated liver cells and its involvement in fibrogenic Smad signalling. Cell Signal 2011;23:683-99.

51 Wiercinska $E$, Wickert $L$, Denecke $B$, et al. Id1 is a critical mediator in TGF-beta-induced transdifferentiation of rat hepatic stellate cells. Hepatology 2006;43:1032-41. 


\section{GUT}

Enhanced expression of BMP6 inhibits hepatic fibrosis in non-alcoholic fatty liver disease

Stephanie Arndt, Eva Wacker, Christoph Dorn, Andreas Koch, Michael Saugspier, Wolfgang E Thasler, Arndt Hartmann, Anja Katrin Bosserhoff and Claus Hellerbrand

Gut 2015 64: 973-981 originally published online July 10, 2014 doi: 10.1136/gutjnl-2014-306968

Updated information and services can be found at:

http://gut.bmj.com/content/64/6/973

\section{These include:}

Supplementary Supplementary material can be found at:

Material http://gut.bmj.com/content/suppl/2014/07/10/gutjnl-2014-306968.DC1

References This article cites 51 articles, 8 of which you can access for free at: http://gut.bmj.com/content/64/6/973\#BIBL

Email alerting Receive free email alerts when new articles cite this article. Sign up in the service box at the top right corner of the online article.

Topic Articles on similar topics can be found in the following collections Collections Nonalcoholic steatosis (127)

\section{Notes}

To request permissions go to:

http://group.bmj.com/group/rights-licensing/permissions

To order reprints go to:

http://journals.bmj.com/cgi/reprintform

To subscribe to BMJ go to:

http://group.bmj.com/subscribe/ 